\title{
Current practice in the use of cone beam computed tomography: a survey of UK dental practices
}

DOI:

10.1038/sj.bdj.2019.49

\section{Document Version}

Accepted author manuscript

Link to publication record in Manchester Research Explorer

\section{Citation for published version (APA):}

Yalda, F., Holroyd, J., Islam, M., Theodorakou, C., \& Horner, K. (2019). Current practice in the use of cone beam computed tomography: a survey of UK dental practices. British Dental Journal.

https://doi.org/10.1038/sj.bdj.2019.49

\section{Published in:}

British Dental Journal

\section{Citing this paper}

Please note that where the full-text provided on Manchester Research Explorer is the Author Accepted Manuscript or Proof version this may differ from the final Published version. If citing, it is advised that you check and use the publisher's definitive version.

\section{General rights}

Copyright and moral rights for the publications made accessible in the Research Explorer are retained by the authors and/or other copyright owners and it is a condition of accessing publications that users recognise and abide by the legal requirements associated with these rights.

\section{Takedown policy}

If you believe that this document breaches copyright please refer to the University of Manchester's Takedown Procedures [http://man.ac.uk/04Y6Bo] or contact uml.scholarlycommunications@manchester.ac.uk providing relevant details, so we can investigate your claim.

\section{OPEN ACCESS}


CURRENT PRACTICE IN THE USE OF CONE BEAM CT: A SURVEY OF UK DENTAL PRACTICES

\author{
${ }^{1}$ Fedil Andraws Yalda \\ 2John Holroyd \\ ${ }^{3}$ Mohammed Islam \\ ${ }^{4}$ Chrysoula Theodorakou \\ ${ }^{5}$ Keith Horner
}

${ }^{1} \mathrm{PhD}$ student, Division of Dentistry, School of Medical Sciences, Faculty of Biology, Medicine and Health, The University of Manchester, Manchester Academic Health Science Centre, Manchester M13 9PL; College of Dentistry, Hawler Medical University, Erbil, Iraq.

${ }^{2}$ Dental X-ray Protection Services, Public Health England, Oak Park Lane, Cookridge, Leeds, LS16 6RW.

${ }^{3}$ Learning Technologist, Faculty of Biology, Medicine and Health, The University of Manchester, Manchester Academic Health Science Centre, Manchester M13 9PL. ${ }^{4}$ Medical Physicist, Christie Medical Physics and Engineering, The Christie NHS Foundation Trust, Manchester Academic Health Science Centre, Manchester M20 4BX; Hon. Research Fellow, Division of Dentistry, School of Medical Sciences, Faculty of Biology, Medicine and Health, The University of Manchester, Manchester Academic Health Science Centre, Manchester M13 9PL.

${ }^{5}$ Professor, Division of Dentistry, School of Medical Sciences, Faculty of Biology, Medicine and Health, The University of Manchester, Manchester Academic Health Science Centre, Manchester M13 9PL. 


\section{ABSTRACT}

\section{Introduction:}

Cone beam computed tomography (CBCT) is increasingly seen in UK dental practices, but there is no published information regarding how it is used. The aim of the study was to obtain such information using a survey.

\section{Materials and Methods:}

A survey on aspects of justification, optimisation and training was distributed to all dental practices having CBCT equipment and a contract with Dental X-ray Protection Services (DXPS).

\section{Results:}

Seventy-one completed surveys were received ( $49 \%$ response rate). A wide range of CBCT equipment was in use. The number of scans reported as being carried out was typically low. One third of respondents had acquired their CBCT machine within the last year. The clinical use was overwhelmingly in adult patients and related to implant dentistry. Small or medium field of view scans were most commonly used. Only $19.7 \%$ of respondents could provide detailed exposure factors. Patient protective shielding for patients was almost never used. The dentist always or mainly performed the CBCT reporting in $88.7 \%$ of practices.

\section{Conclusions:}

The survey provided no evidence of excessive use of CBCT in UK dental practices. The typical reported use was of small field of view scans for implant dentistry. Only $8.4 \%$ of practices performed examinations on children and young people. Some concerns were raised regarding respondents' knowledge about exposure factors and the clinical evaluation of images. 
IN BRIEF

- Presents an overview of the current use of CBCT in UK dental practices

- Suggests that most CBCT examinations are small field of view scans for adult patients in the context of implant dentistry

- Raises some questions about knowledge of exposure factors and the training for clinical evaluation of $\mathrm{CBCT}$ images 


\section{INTRODUCTION}

Cone beam Computed Tomography (CBCT) has been commercially available to dentists since the start of the century. Initially, there were only a small number of СBCT machines on the market, but by 2013 this had risen to $43 .{ }^{1}$ Today it is likely that many more systems are available, particularly as hybrid panoramic/ cephalometric/ CBCT units have become more prevalent. CBCT offers crosssectional imaging at potentially high geometric accuracy, a feature of particular interest to practitioners planning dental implant treatment. Its use has, however, expanded to potential applications in many branches of dentistry. ${ }^{2}$ Because of the typically higher radiation doses compared with traditional dental radiography, there have been concerns expressed about proliferation of its use, particularly with regard to paediatric applications.

Unlike in some other countries, there is no legal requirement for national registration of dental CBCT equipment in the UK. The evidence for the growth of CBCT in UK primary dental care facilities is therefore largely indirect, obtained from anecdotal information from manufacturers and dental equipment installers. There is little independent evidence for numbers of $\mathrm{CBCT}$ equipment in dental practices or, in the context of justification, the clinical purposes for which it is being used. Although guidelines highlight the need for optimisation of exposures when using $\mathrm{CBCT},{ }^{2,3}$ the literature in this area is small and is hard to translate beyond the context in which the research was carried out. Only two surveys are available from the UK. ${ }^{4,5}$ One of these looked only at paediatric use and the other was made up predominantly of children and younger adults; both were NHS-hospital based. They each highlighted deficiencies in information regarding justification and optimisation of CBCT in clinical 
practice. Their findings are, however, of limited relevance to "high street" dental practices, many of which are independent of the NHS. Dentists in UK primary care "self-justifyrefer" their X-ray examinations, meaning that the referral, justification and authorisation components of undertaking X-ray examinations are usually performed by the same person. Unlike hospitals, dental practices have limited access to medical physics support and rarely employ radiographers to undertake the examinations. Two detailed survey reports exist from Norway and Sweden ${ }^{6,7}$ and two very limited surveys from Turkey, ${ }^{8,9}$ but the practice of dentistry varies internationally and the findings are not transferable to the UK. It is clear that the evidence for use of CBCT in primary dental care is extremely limited internationally and none is available for the UK. There would, therefore, be value in obtaining information about CBCT use in UK dental practice related to aspects of justification, optimisation and training.

The research question underlying this survey was therefore "what is the profile of use of $\mathrm{CBCT}$ in primary care dental practices in the UK, with regard to justification, optimisation and training?" The objective was to obtain an overview of the use of CBCT in primary care dentistry in the UK, by means of a survey, taking special note of paediatric use. 


\section{MATERIALS AND METHODS}

This study was a collaboration between the University of Manchester and Dental Xray Protection Services (DXPS) of Public Health England (PHE; https://www.pheprotectionservices.org.uk/dxps). DXPS agreed to collaborate as the study would provide them with information about the clinical uses of dental CBCT that is not collected as part of their Radiation Protection Adviser/Medical Physics Expert (RPA/MPE) role for dentists. DXPS acted as the "middle man" for the survey, sending out emails and posts to the dental practices on behalf of the Universitybased researchers to ensure the anonymity of their customers to the academic researchers.

\section{Research tool}

A mixed-mode survey was used, offering surveyed dental practices a choice of responding on paper or online. The survey questionnaire content was new, but included some questions inspired by previously published surveys. ${ }^{6,7}$ The content was developed after two iterations of a pilot survey, each conducted on ten dental practices selected randomly from the sample frame by DXPS.

The definitive survey consisted of 28 items (23 main items, four follow-up questions and one optional item at the end), listed in Table 1 and in the online format using web-based questionnaire SelectSurvey.NET (v4.075.003, Classaps, Kansas City, MO, USA; http://classapps.com) at: 


\section{$\underline{\text { Sample selection }}$}

DXPS provides a Radiation Protection Adviser/Medical Physics Expert (RPA/MPE) service for dental practices, including for those with $\mathrm{CBCT}$ equipment. At the time of conducting the survey, 145 dental practices with CBCT equipment were contracted with DXPS and formed the sample frame for the survey. The total number of primary care dental practices in the UK with CBCT is unknown but has been estimated at around 950 by one of the authors $(\mathrm{JH})$. Multiple other providers of RPA/MPE services exist, including local hospital Medical Physics departments and independent radiation protection companies, but numbers of dental practices using their services and their identities are often commercially confidential. As it was impractical to determine an exact potential sample frame for the survey, a decision was taken to conduct the survey on all 145 dental practices registered with DXPS.

\section{Survey administration}

The anonymity of the dental practices surveyed in the study was maintained by allocation to each practice of a study number by DXPS and by all communications to the practices coming from or via DXPS. Invitations to participate in the survey were sent at the same time in two ways. First, DXPS sent an invitation email to the contact person in each dental practice, containing the link to the online survey. Secondly, DXPS sent a package by post containing an invitation letter, a paper version of the survey, a pre-paid envelope for returning the questionnaire and also a £20 gift voucher. The invitation email and letter were signed by all the authors and included their job titles. As most dental practices were likely to have multiple dentists and possibly multiple operators of CBCT equipment, the email/postal recipient was asked to select the most appropriate person to respond to the questionnaire. Respondents 
were told to use whichever format, online or paper, to complete the survey that they preferred.

Responses to the online survey were monitored by an IT specialist at the University of Manchester. Responses to the paper survey were monitored by the first author. The survey was started on 05/12/2017. Two email reminders about the survey were sent. The first reminder was sent to non-responders one week after the start of survey, and a second reminder was sent after one further week. The closure date for the definitive survey was set at 31/01/2018.

This study did not constitute research as defined by the NHS using the Health Research Authority Decision Tool (http://www.hra-decisiontools.org.uk/research/) and that NHS ethical approval was not required. Direct email contact with the HRA Queries Line Advisers confirmed that the survey was classifiable as a service evaluation and that NHS ethics approval was not needed. University advice was also sought regarding the need for ethical approval for the study at the protocol writing stage. The guidance was that research involving interviews or surveys with participants on subjects deemed to be within their professional competence, in which the researcher is not asking the participant to reveal personal, confidential or sensitive information and the subject matter is well within the professional competence of the interviewee, does not usually require ethical review. Specific confirmation was received from the University's Research Governance, Ethics and Integrity Officer (Ethics). 


\section{$\underline{\text { Analysis }}$}

Survey responses were entered into a Microsoft Excel 2016 worksheet to calculate frequencies of responses to each question. Statistical analysis was performed by using the Statistical Package for the Social Sciences (SPSS) version 23 (2015) (SPSS IBM Corporation, Armonk, New York, USA). A two-sided chi-squared test was used to examine the significance of any association between the dichotomised answers to the questions regarding the most commonly used Field of View (FOV) and reporting being performed by the dentist rather than involving a radiologist at least in some cases. For this, the FOV answers were dichotomised into "smallest + medium" and "large + full head" and the reporting operator identity answers dichotomised into "always the dentist who requested the scan" with all other combinations combined.

An assessment of non-response bias was carried out by comparing the responses to main survey questions, excluding the four follow-up questions and two optional items at the end, made by "early responders" (those who responded to the first notification of the survey) and "late responders" (those who responded after reminder communications), as described by Groves. ${ }^{10}$ The null hypothesis was that there would be no significant differences between the two groups in responses to the 23 main item questions (Table 1). Two-sided chi-square tests were planned to be performed for each main question to assess any association between responder group (early or late) and the dichotomised answers to each of these questions except question 3. Dichotomisation of answers to all except question 3 was carried out at a level closest to the median point of the distribution of answers. For question 
3, answers were grouped into four sub-groups according to the CBCT equipment manufacturers.

For all statistical analyses, an alpha of 0.05 was used as the cut-off for significance. 


\section{RESULTS}

Of the 145 dental practices in the sample frame, 74 responses were received. One of these was a University dental clinic and, although a completed questionnaire was received, it was in retrospect considered by the researchers as ineligible as the context was not in line with a general dental practice. Two respondents returned blank paper questionnaires, declining to participate in the survey as they thought that the survey was not anonymous. Thus, the total number of eligible participating dental practices was 144 and the number providing eligible completed surveys was 71 , giving a response rate of $49 \%$. Of these, $54(76 \%)$ returned questionnaires in paper format. One mobile dental CBCT clinic participated in this survey, so some categories of questions in the survey were not applicable for them. Consequently, the total number of responses to each main questionnaire item varied between 70 and 71.

Responses to survey items are presented below, in the order of the questions asked in the survey (Table 1). The number of dentists working in each dental practice ranged from one to 26 with a modal number of four dentists (Table 2). Fewer dentists in the same practices were reported as using CBCT frequently, with 52 dental practices $(73.2 \%)$ reporting only one or two dentists in response to this question.

Twenty-six different CBCT scanner models from eight manufacturers were used in the dental practices (Fig.1). No respondents had more than one CBCT scanner in their dental practice. Sirona and Carestream (including Kodak) were most commonly represented manufacturers, each seen in 19 (26.7\%) dental practices, followed by Vatech (including E-Woo) in $16(22.5 \%)$ dental practices. The Sirona Orthophos 
XG3D was the most frequently used CBCT model (14 dental practices, 19.7\%), followed by the Carestream CS8100 3D (seven dental practices, 9.9\%). It should be noted that the Carestream CS 9000, Kodak 9000 3D and Kodak k9000 C 3D are essentially the same machine carrying a different model name.

Table 3 shows that the great majority of respondents (54 dental practices, $76 \%$ ) had a CBCT scanner in their practices for less than five years. Notably, one third of the dental practices had acquired their CBCT machine within the last year. While 16 (22.5\%) of dental practices used the CBCT machine only for their own patients, the remainder accepted external referrals. The mobile CBCT provider was an exception for this question as they only accept external referrals.

As shown in Fig. 2, a small FOV, defined as having a size sufficient to image one tooth or a few teeth and their supporting bone, and medium FOV (one jaw or a full quadrant) were the most commonly used FOVs (51 clinics, 71.8\%). Conventional radiographs (intra-oral and/or panoramic) of patients were always available to dentists at some point before deciding to perform CBCT examinations in 26 (36.6\%) of dental practices and for the majority of patients in $34(47.9 \%)$ of practices. Six respondents $(8.4 \%)$ said that they never had them available and five $(7 \%)$ reported that they had them only in a minority of cases.

A majority of the clinics estimated that they scanned 1-10 adult patients per month. Only $11.2 \%$ of responders scanned more than 20 adult patients per month (Table 4). The commonest single reason for $\mathrm{CBCT}$ examinations in adult patients among the responses was implant planning (62 responses). Only one responder answered 
endodontic assessment. Despite asking for the commonest reason (singular), eight respondents answered with combinations of reasons. It is noteworthy that for these, implant planning was part of the answer for all. The indications cited in combination were impacted teeth assessment (four responses), endodontic assessment (three), and orthodontic diagnosis in one response. So the number of responses citing implant planning, either alone or in combination, as the commonest reason for the scans in adult patients was 70 out of the 71 completed and eligible surveys.

The overwhelming majority of respondents could not provide the detailed exposure factor settings for adult patients. Most $(51,71.8 \%)$ reported that they used preprogrammed settings. Fourteen (19.7\%) provided the exposure details. Of the remaining survey responses, five answered that they had an automatic exposure control (AEC), while one respondent left the answer to this question blank.

The majority of dental practices (61 responses, $91.6 \%$ ) did not perform CBCT on children. Of the six respondents who did use CBCT in this age group, the commonest reasons given were impacted tooth localisation (two responses), orthodontic diagnosis (two responses), and one response each for "implant planning" and "use as panorama for diagnosis when patient cannot tolerate bite-wings". Only one of the six dental practices specified exact exposure values, showing a reduction in exposure time and tube current for children compared with the values they had specified for adult patients. The other five practices simply reported that they used pre-programmed settings. 
Answers to the request for details of the underlying clinical reason for carrying out CBCT examination for each of the last five patients scanned in the dental practice are given in Table 5. Implant planning and other implant-related purposes dominated the responses, being the primary reason for $84.7 \%$ of scans. For 15 patients, combinations of clinical reasons were cited. If endodontic diagnosis, root resorption and periapical pathosis diagnosis are combined into one broad "endodontic" category, these were given as a reason, either primary or secondary, for the scan for 30 patients $(8.5 \%)$.

The overwhelming majority of dental practices (68 responses, $96 \%$ ) did not use a lead or lead equivalent thyroid shield. This question was unanswered by one respondent. Two dental practices reported that they used thyroid shields, with one of these saying that they used it "sometimes", explaining that this referred to mandible exposures which could include the thyroid and if the shielding would not interfere with the image. For lead or lead equivalent aprons, an identical proportion of respondents reported that they did not use them (68 responses, 96\%) and there was one missing response. Of the remaining two dental practices who answered the question, one confirmed that they used a lead apron and the other responded that they used one "sometimes" but without specifying the situations.

Table 6 shows the responses to the question about who reports the CBCT scans. By combining categories in the Table, it can be seen that the dentist always or mainly performed the CBCT reporting in 63 dental practices (88.7\%). The majority $(57.5 \%)$ of responders who had stated that larger fields of view were the most commonly used also reported that the dentist always reported the scans, whereas $53 \%$ of those 
who most commonly used smaller FOVs responded in this way. There was no significant difference in these proportions of larger and smaller FOVs interpreted always by the dentist $(p=0.81)$. In terms of training for the task of reporting (Table 7$)$, nobody stated solely that the dentist had enough information to report CBCT images without additional training, although one included that statement in a combined reply. Assessment of non-response bias planned for the 23 main survey questions (Table 1) was not possible because of low numbers responding to the question, notably for paediatric-related questions (12 to14) or because of answers being overwhelmingly the same (e.g. in relation to lead shielding (questions 20 and 22). For all the questions for which a statistical comparison was feasible, there were no significant differences between early and late responders except in the case of question 5 ("For how many years has СВCT been available in practice?"). For this question, if the dichotomisation of answers was made at the $\leq 3$ years/ $>3$ years level there was a significantly greater proportion of late responders in the group who had had CBCT available for more than three years $(p=0.016)$. It should be noted, however, that changing the level for dichotomisation to the $\leq 2$ years/ $>2$ years gave a nonsignificant result. 


\section{DISCUSSION}

When conducting surveys, it is important to consider the four main sources of potential error: coverage error, sampling error, non-response error and measurement error. ${ }^{10,11}$ In terms of coverage, an accurate knowledge about the numbers of dentists in the UK with CBCT equipment is not obtainable due to the absence of any information source. This is in contrast with the comparable studies in Scandinavia ${ }^{6,7}$ as, unlike in the UK, there is a requirement to register the use of CBCT equipment in Sweden and Norway. Public Health England DXPS is probably the largest supplier of RPA/MPE services to dentists in the UK but there are others, including local hospital medical physics departments, which individually may only advise a handful of dental practices. In retrospect it would have been useful to involve all identifiable suppliers, although there is no obvious reason why dental practices using DXPS would give a biased sample relative to the larger potential sample frame of practices using all RPA/MPE providers. Indeed, as a national provider, DXPS probably provides a better geographical coverage to alternative providers. The current survey certainly is superior in coverage to the two Turkish studies ${ }^{8,9}$ which used convenience samples of conference attenders, a strategy which has been strongly criticised because conference attenders may not be representative of the wider population. We chose to survey the complete number of dental practices using the services of DXPS rather than a random sample of these. Although surveying the entire available population has been criticised in the past on the basis that sampling of the population can be more cost-effective, such criticisms tend to be aimed at very large studies in which a smaller sample might be easier to achieve a high response rate by a more personalised approach. In our case, the 145 dental practices using DXPS was already small and we felt that this was manageable. Furthermore, the 
option of a personalised attempt at obtaining responses from dental practices, e.g. using telephone contact, was impossible because of the requirement for anonymity of the dental practices.

Despite considerable time and effort expended in developing the survey, including piloting, offering a choice of formats for responses and a financial incentive, the response rate was very disappointing. There are numerous possible reasons for poor response that are common to all surveys, but in this particular case it is possible that dentists might be unsure on the governance, regulation and legal implications relating to this relatively new imaging modality and be a little hesitant in providing information. The concern must always be that a low response rate leads to non-response error, i.e. that the non-responders are different to responders in terms of the items investigated by the survey. As this is an unknown which cannot be answered with certainty, strategies have been suggested to measure the risk of nonresponse bias. Groves described some methods of assessing the possibility of nonresponse bias, but only one of these was practicable in our case. ${ }^{10}$ The method chosen to assess non-response bias risk is based on the assumption that late responders to a survey are likely to be similar to non-responders. ${ }^{10}$ Our assessment of this showed no significant differences in question responses except for one, the length of time for which the CBCT scanner had been available in the practice. It is possible to suggest an explanation for this; newer users of the CBCT technology might well have a greater interest in it than those who are accustomed to it and keener to take part in a survey. While this finding is interesting, the lack of a significant difference in the answers to other questions between early and late responders provides some reassurance about non-response bias. Overall, however, 
the possibility of non-response bias cannot be confidently excluded by any method, including this one, and our requirement for an anonymous survey prevented alternative efforts at improving response and investigating the risk of non-responder bias. Nonetheless, it is important to note that a low response rate does not mean a high risk of non-response bias; similarly, a high response rate does not exclude significant non-response bias. ${ }^{11}$

Recent data ${ }^{12}$ on the numbers of dentists in the UK and the total number of dental practices (as determined by VAT and/or PAYE registration in Standard Industrial Classification 8623) as gives a crude estimated average of three to four dentists per dental practice, a figure which is broadly in keeping with the survey results (Table 2). Dentists regularly using CBCT typically in the dental practices were fewer, probably because only a proportion of dentists would undertake implant dentistry, the predominant clinical task for which CBCT was being used.

In 2013, one international review of CBCT found 43 CBCT scanners were available on the market, ${ }^{1}$ differing remarkably in their technical specifications. This figure is likely to be greater now, as new models have been launched but old scanners continue to be used. Our survey identified 26 scanner models in the responding dental practices, with three manufacturers featuring prominently. A survey in Norway and Sweden ${ }^{7}$ also reported a wide range in equipment manufacturers, although it is notable that the dominant manufacturer in Sweden hardly featured in our UK survey. Such differences reflect multiple factors, purchase cost, manufacturers' marketing efforts in different countries, along with servicing and maintenance support. The view of the authors, based on wide reading of the 
research literature related to diagnostic efficacy, is that the profile of CBCT scanners identified in our survey is very different to those commonly used in research studies and which inform guidelines on diagnostic usefulness. As the technical specifications of $\mathrm{CBCT}$ equipment are very variable, it is interesting to hypothesise that the evidence for diagnostic efficacy using CBCT on which current guidelines are based may not be applicable to the equipment commonly used in UK dental practices.

The data in Table 3 suggest that rate of acquisition of CBCT equipment is increasing annually. This would not be surprising when a successful new technology is introduced, but it is likely to continue as several manufacturers now offer "hybrid" panoramic/ cephalometric/ CBCT equipment at relatively low costs compared with dedicated CBCT scanners. As old panoramic equipment requires replacement, it is likely that many dental practices will use the opportunity to upgrade to a one that includes a СВСТ option. One interesting finding was that about three quarters of dental practices were open to external referrals for $\mathrm{CBCT}$. As CBCT equipment can be very expensive, it seems reasonable to try to generate additional income in this way, although this does incur legal responsibilities related to referral, justification of examinations and reporting for the dental practice. ${ }^{13}$ In particular, the practice receiving referrals from others must ensure that the referrer has provided adequate clinical information to permit the justification process to be performed properly. It $\underline{\text { might also be helpful (although not absolutely required) for the practice receiving }}$ referrals to provide referral guidelines to potential referrers. The dentist receiving the referral must justify the examination and authorisation must also be carried out. Clinical evaluation of the images needs to be performed and the responsibility lies in 
the first instance with the practice undertaking the CBCT examination, although it is common for this responsibility to be transferred to the referring practice by a formal service level agreement.

The FOV is a major determinant of radiation dose in $\mathrm{CBCT}^{2,3}$ and guidelines emphasise the importance of using the smallest FOV compatible with the clinical task. It was therefore reassuring to find that the "smallest", or the "medium", FOVs were the most commonly used. This was also the finding of the Scandinavian study ${ }^{7}$ and could indicate that guidelines on good practice might have been effective. Reducing the FOV not only reduces radiation dose, but may also improve image quality through reduction in X-ray scatter and limit the volume of information to be reported. A less reassuring finding from the responding dental practices was that conventional radiographs were not always available to dentists before deciding to perform CBCT examinations. Almost all guidelines, reviewed by Horner et al.., ${ }^{13}{ }^{14}$ are agreed on a fundamental principle that CBCT could be justified when conventional radiographs do not answer the diagnostic question for which imaging is required. There are, however, some reasons why the results in the survey are not in keeping with this. For referred patients, it is unlikely that the referring dental practice would send their conventional radiographs to the practice undertaking the scan; instead, necessary information about conventional radiographic results might have been sent with the referral. Secondly, for implant planning, which was reported as being the main reason for performing $\mathrm{CBCT}$, there is perhaps some argument for going straight for cross-sectional imaging, a position recognised by German guidelines. ${ }^{13}-{ }^{14}$ However, this view can be countered by recognising that initial conventional radiography of a proposed implant site might reveal an abnormality 
(e.g. retained root or unerupted tooth) that would contraindicate an implant until after it had been treated; this might avoid the need for a second scan.

One finding of particular note is the small number of patients reported as being scanned in the dental practices. Most respondents said that they scanned no more than ten adult patients per month and only $11.2 \%$ reported scanning more than 20 adults each month. Although three quarters of responding practices were open to external referrals, it was not leading to high levels of patient throughput. This raises questions over the cost effectiveness of having a CBCT scanning facility within a dental practice, although it is important to note that some of the equipment used is of the "hybrid" type and would also be used for panoramic and possibly cephalometric radiography. The low level of use seen in our study agrees with the situation reported in a Norwegian survey, in which $72 \%$ of clinics performed an average of four or fewer CBCT examinations per week, with specialist clinics averaging five to ten per week. ${ }^{6}$ In terms of economic effectiveness, referral for CBCT to specialised centres or the innovative provision of a mobile $\mathrm{CBCT}$ service, as is the case for one of the responders to the survey, may be a more sensible way of delivering this service.

Previous surveys in Sweden, Norway and Turkey have reported that implant treatment planning was the most common indication for $\mathrm{CBCT}$ examination. ${ }^{6,7,8}$ Our survey also found this to be the case for adult patients, but overwhelmingly so. Horner et al. reported that although the majority of guidelines state that crosssectional imaging is indicated in selected cases as part of planning dental implants, a few guidelines propose that it is always essential. ${ }^{14}$ CBCT for implant planning is 
probably the most straightforward of indications to justify, particularly as it would be unusual to perform implant dentistry in those under 18 years, when radiation risk is higher and justification a more exacting task. Uses of CBCT for other diagnostic purposes were few. This is unlike the findings in the surveys from other countries, where impacted tooth assessment, "jaw pathology" and pain-related problems each contributed substantial proportions of the reasons for using СВСТ. ${ }^{7}$ In the Turkish survey by Dölekoğlu et al., ${ }^{8}$ diagnosis of cysts/tumours was second to implant planning as the commonest reason for prescribing CBCT. The other Turkish survey, which was solely of endodontists, found that "cysts/ tumours" was the most common reason for $\mathrm{CBCT}$ being used. ${ }^{9}$

An important part of the objective of the survey was to take special note of paediatric use of $\mathrm{CBCT}$, bearing in mind the radiation dose of $\mathrm{CBCT}$ and the greater risk of stochastic effects in children and young people. If our survey is representative of the national picture, then it is evident that there is very limited use in this age group and suggests that concerns over excessive use in children may not be real, although isolated exceptions could easily exist. In fact, the reported use was so low that no other useful information can be gleaned from the results. It is likely, however, that specialist orthodontic practices with CBCT equipment might behave differently and it would be valuable in the future to look specifically at such practices. In UK Dental Hospital-based practice, Hidalgo-Rivas et al. found that $13.7 \%$ of all CBCT examinations over a 24-month period were conducted on children and young people, most commonly for localisation of unerupted teeth in the anterior maxilla and particularly in the context of detection of root resorption of adjacent teeth. ${ }^{5}$ Even this is still a modest level of use compared with adults. One answer included as a 
commonest reason for performing $\mathrm{CBCT}$ in this age group, "use as panorama for diagnosis when patient cannot tolerate bite-wings", does, however, cause concern as all current guidelines that consider the use of $\mathrm{CBCT}$ in caries diagnosis state that it should not be used for this purpose. ${ }^{13}-14$

Apart from FOV, the main determinant of radiation dose to the patients undergoing $\mathrm{CBCT}$ are the exposure settings $(\mathrm{kV}, \mathrm{mAs})$. It was apparent that most responders could not provide this information, with just under $20 \%$ able to a give detailed response. The "pre-programmed settings" used by the majority presumably represent a "standard male", "standard female" type of setting. How many settings of this kind are available on a scanner and how they are used by the operator will vary widely, but they may not offer the opportunity for individualised exposures to be set as when specific exposure settings can be altered. The lack of awareness of the exposure settings may also indicate that the pre-programmed settings may not be appropriate for the relevant patient size if the practice has not undertaken any optimisation work to review 'factory default' exposure settings. Interestingly, none of the five respondents who specified that they used an AEC had CBCT equipment with that specification. In these cases, the best that can be assumed is that the term AEC was confused with pre-programmed settings. We had hoped to identify whether exposure parameters were reduced for $\mathrm{CBCT}$ examinations of children and young people, but the limited number of dental practices performing scans in this age group means that no generalisable findings can be identified. Overall, the findings in this survey showed a lack of awareness of the exposures used and a reliance on pre-programmed settings. This appears to be in contrast to previous 
survey in Sweden and Norway, where the majority of respondents were able to specify the technical parameters they could alter. $^{7}$

Almost all dental practices complied with current guidelines in the $\mathrm{UK}^{3}$ on use of CBCT equipment, which state that there is no need on radiation protection grounds for patients to wear lead aprons for CBCT examinations. The UK guidance on thyroid shielding is less definitive, recommending a case-by-case approach with advice from the MPE based on evidence that thyroid shielding can lower effective dose to patients when larger FOVs are used or when the FOV is close to the neck. Since the UK guidance was written, evidence for the efficacy of thyroid shielding has accumulated and it may be appropriate to suggest that it is used for CBCT, although artefacts will be produced when the shield overlaps the primary beam and potential problems exist for AEC operation. MPE advice is therefore still appropriate.

According to dentists in a previous survey in Norway, ${ }^{6}$ the biggest challenges when acquiring $\mathrm{CBCT}$ in dental practice is learning how to use the equipment and interpret the images. A curriculum for additional training has been defined in the UK and also at a European level. ${ }^{3,14}-{ }^{15}$ The UK curriculum specifies that the training described was intended to equip the dentist for interpretation of scans of the dento-alveolar region only and did not include interpretation of the skull base, temporal bones, neck and spine that might be partially included on larger FOV scans. ${ }^{3}$ It is pertinent to note that there is a requirement for a specialist radiologist's report in Sweden and Norway for scans not limited to the dento-alveolar region. ${ }^{6,7}$ In our survey, a large majority of respondents stated that in their practices the dentists reported all or most of the CBCT examinations and the data suggest that this was the case regardless of 
FOV size. Although respondents had undergone training (Table 7) our survey did not explore the nature of it in sufficient detail for any assessment of its adequacy.

However, the lonising Radiation (Medical Exposure) Regulations ${ }^{15}$-Regulations ${ }^{13}$ require that those with an operator role (including clinical evaluation) are adequately trained. It is notable that $12.7 \%$ of respondents stated that they only had training from the installers of the X-ray equipment; it is to be hoped that the installers employed a suitably trained clinician to train the dentists.

The relationship with the NHS of the dental practices surveyed in this study is unknown. Some may have been independent while some may be providing a mix of NHS and private dentistry. It is very unlikely that any practice only provided NHS treatment, bearing in mind that the overwhelming use of the CBCT scanners was for implant dentistry. A question was not included in the survey on this as it was not of primary interest and would have unnecessarily lengthened the content. The regulations relating to the use of ionising radiation ${ }^{13,16}$ apply equally to independent practices and those providing NHS commissioned services. The organisations in the four home nations that monitor, inspect and regulate services with regard to the use of ionising radiation are the same for independent practices. It seems unlikely to us that there would be any significant difference in study findings according to whether practices performed NHS-commissioned services or not. 


\section{CONCLUSIONS}

This survey provides new information about the use of CBCT in UK dental practices. It was reassuring to find that there was no evidence to suggest excessive use of CBCT and, if anything, equipment typically appears to be only lightly used and its cost-effectiveness could be questioned. If a typical CBCT examination in UK dental practice can be described, it would be a small or medium FOV scan in an adult patient taken for implant dentistry. Some concerns, however, arise from the survey regarding the knowledge about exposure factors used for $\mathrm{CBCT}$ and in relation to aspects of interpretation of images. There is no suggestion of frequent use in children and young people, with a reported frequency in the survey that was too low to analyse. Future surveys might usefully be focused on specialist practices which see a proportionally greater number of children and young people, bearing in mind the importance of radiation protection in these age groups. 


\section{DECLARATION OF INTERESTS}

Dental X-ray Protection Services (Public Health England) received funding from the University of Manchester to cover administration costs associated with their participation in the study. The authors declare no conflicts of interest. 


\section{ACKNOWLEDGMENTS}

Thanks are due to all the respondents to the survey for their time. 


\section{REFERENCES}

1. Nemtoi A, Czink C, Haba D, Gahleitner A. Cone beam CT: a current overview of devices. Dentomaxillofac Radiol 2013; 42: 20120443.

2. European Commission. 2012. Radiation protection No 172. Cone beam CT for dental and maxillofacial radiology (evidence based guidelines). Luxembourg: European Commission Directorate-General for Energy. Online information available at https://ec.europa.eu/energy/sites/ener/files/documents/172.pdf (accessed March 2018)

3. HPA Working party on Dental Cone Beam CT Equipment. Guidance on the safe use of dental cone beam CT (computed tomography) equipment. Chilton: Health Protection Agency, 2010, publication no. HPA-CRCE-010.

4. Dobbyn LM, Morrison JF, Brocklebank LM, Chung LL. A survey of the first 6 years of experience with cone beam CT scanning in a teaching hospital orthodontic department. J Orthod 2013; 40: 14-21.

5. Hidalgo-Rivas JA, Theodorakou C, Carmichael F, Murray B, Payne M, Horner K. Use of cone beam CT in children and young people in three United Kingdom dental hospitals. Int J Paediatr Dent 2014; 24: 336-348. 
6. Hol C, Hellén-Halme K, Torgersen G, Nilsson M, Møystad A. How do dentists use CBCT in dental clinics? A Norwegian nationwide survey. Acta Odontol Scand 2015; 73: 195-201.

7. Strindberg JE, Hol C, Torgersen G, et al. Comparison of Swedish and Norwegian Use of Cone-Beam Computed Tomography: a Questionnaire Study. J Oral Maxillofac Res 2015; 6: e2.

8. Dölekoğlu S, Fişekçioğlu E, İlgüy M, İlgüy D. The usage of digital radiography and cone beam computed tomography among Turkish dentists. Dentomaxillofac Radiol 2011; 40: 379-352.

9. Yalcinkaya SE, Berker YG, Peker S, Basturk FB. Knowledge and attitudes of Turkish endodontists towards digital radiology and cone beam computed tomography. Niger J Clin Pract 2014; 17: 471-478.

10. Groves RM. Survey errors and survey costs. $2^{\text {nd }}$ ed. Hoboken, NJ: John Wiley \& Sons, 2004.

11. Shelley AM, Brunton P, Horner K. Questionnaire surveys of dentists on radiology. Dentomaxillofac Radiol 2012; 41: 267-75.

12. Office for National Statistics. Freedom of information request FOI 3748. Online information available at 
https://www.ons.gov.uk/aboutus/transparencyandgovernance/freedomofinformationf oi/numberofdentistsanddentalpracticesintheuk (accessed May 2018).

1513. The lonising Radiation (Medical Exposure) Regulations. London: HMSO, 2017. Statutory Instrument no. 1322.

1314. Horner K, O'Malley L, Taylor K, Glenny AM. Guidelines for clinical use of CBCT: a review. Dentomaxillofac Radiol 2015; 44: 20140225.

1415. Brown J, Jacobs R, Levring Jäghagen $\mathrm{E}$, et al. Basic training requirements for the use of dental CBCT by dentists: a position paper prepared by the European Academy of DentoMaxilloFacial Radiology. Dentomaxillofac Radiol 2014; 43: 20130291.

15. The lonising Radiation (Medical Exposure) Regulations. London: HMSO, 2017. Staturystument no. 1322

16. The lonising Radiations Regulations. London: HMSO, 2017. Statutory Instrument no. 1075. 


\section{FIGURE LEGENDS}

Fig.1: Types of CBCT scanner used by the 71 respondents to the survey. No respondent had more than one scanner in their dental practice. The colour of the bars represents individual manufacturers.

Fig. 2: The number of responses received in the survey regarding the most commonly used field of view in the dental practice. 